\title{
Development of a Smart Grid Simulation Environment, Part I: Project of the Electrical Devices Simulator
}

\author{
Jônatas Boás Leite · José Roberto Sanches Mantovani
}

Received: 26 September 2014 / Accepted: 30 September 2014 / Published online: 17 October 2014

(C) Brazilian Society for Automatics-SBA 2014

\begin{abstract}
A smart grid is an electric power system with high levels of automation, dispersed generation, intelligent monitoring and control; however, because the distribution networks in operation today do not have these characteristics, studies concerning the control, planning and operation of the smart grid are difficult to perform. To overcome these practical difficulties, studies investigating the smart grid can be conducted in computational environments that are able to reproduce meticulously the electrical and communicational behaviors expected by the smart grid. Therefore, in this paper, the development of a platform to simulate the advanced distribution management system from a reference model for smart grid, which has seven layers, is proposed. Part I of this paper is dedicated to formulating mathematically the three lower layers that make up the electrical devices simulator. The analysis of the results revealed the capability of the simulator to generate scenarios with a wide range of power demand that is ideal for developing new operation, planning and control tools for a smart grid.
\end{abstract}

Keywords Smart grid Advanced distribution management system (DMS) · Reference model

\section{Introduction}

A smart grid is characterized by two-way flows of electricity and information enabled by the integration of the power grid with information and communications technologies and

J. B. Leite (更) · J. R. S. Mantovani

Electrical Engineering Department, UNESP/FEIS, Ilha Solteira, São Paulo, Brazil

e-mail: jonatasboasleite@gmail.com

J. R. S. Mantovani

e-mail: mant@dee.feis.unesp.br by the use of the demand response management that coordinates the distributed energy resources, the electrical loads and the charging of plug-in electric vehicles (Deilami et al. 2011). Real-time active operations, such as self-healing and plug-and-play support, are also expected features of a smart grid, which enable the active participation of the end customers in the energy markets and the creation of new products and services. In this way, the energy reliability, quality and efficiency can be maximized. Therefore, the major challenges to the smart grid researchers are related to smart devices, communication systems, cybersecurity, data management and software application.

Smart meters are important smart devices in the smart grid structure because smart meters support the automatic reading operations, facilitate the management of the home appliances and are essential components of the advanced metering infrastructure (AMI) (Cho and Yamazaki 2009). The AMI communication network is hierarchical and has many smart meters arranged through the neighborhood area networks at the bottom of the hierarchy. The units of the data aggregation receive the data from the smart meters and send them, via a wide-area network, to the meter data management system (MDMS) that is located at the top of the hierarchy. The MDMS data can be used to forecast the power grid loading and to help with the decisions of the protection system. Moreover, the metering data are processed with market data to perform operations such as the economic dispatch (Niyato and Wang 2012). The same optimistic results verified in the smart devices area have been obtained in the communication systems area.

The typical SCADA communication system has a low data rate, which is not suitable for smart grid operations; in contrast, a wide-area monitoring system (WAMS) uses a highspeed Internet communication system and represents the next generation of the sensor network that is suitable for the smart 
grid architecture. The WAMS can monitor the power system infrastructure and detect mechanical failures, such as conductor failure, tower collapse, hot spots and extreme mechanical conditions (Qiu et al. 2011; Leon et al. 2007). The phasor measurement unit (PMU) is the most important component of a WAMS and provides time-synchronized data of the power system that are useful to the real-time operations. In essence, the PMU was created to measure the phasors of voltage and current, the frequency, and the real/reactive power in realtime with global positioning system timer tagging. Another important aspect of a smart grid is data safety.

Several smart grid devices use an Internet connection to exchange information; thus, cyber security becomes a crucial resource that ensures the integrity and resiliency of smart grid operations. An Internet-based load altering attack, for example, might be a common cyber-attack that damages the power system by the circuit overflow or disruption of the balance between power supply and demand. This vulnerability can exist because the system operator transmits messages of monitoring and control to the smart meters or to the home energy management systems via the Internet-hosted servers. In this way, a set of defense mechanisms, such as the protection of command and price signals, protection of smart meters and data centers, attack detection and learning demand patterns, load shedding, and load relocating, must help in blocking or minimizing Internet-based attacks (Palensky and Dietrich 2011; Mohsenian-Rad and Leon-Garcia 2011). Cyber security must be present in all levels of the smart grid structure, primarily in the management systems.

In the top of the smart grid structure, there are the data management and the software applications representing the administrative core of the DMS that require a distributed architecture, provisioning of the intelligent service, ability to address complex and dynamics events and support to the several energy sources, such as the new-renewable energy and distributed energy, to coordinate conventional production units with nonconventional units to form the microgrid (Byun et al. 2011; Kanchev et al. 2011). A multiplatform architecture applied on the DMS must enable the interfacing with other applications, such as the geographic information system, via the standardized Web-based user interfaces. Consequently, two or more networks, systems, devices, applications, or components can exchange information between them and use it (Lightner and Widergren 2010). Other resources, such as data filtering and visualization, should enable rapid analysis of the network conditions and improve the decision-making process, for example, the tools of dynamic coloring of the network graph improves the answer in stressful situations (Singh et al. 1998).

The challenges related to the smart grid are under investigation, and solutions have been proposed; however, currently, only a few devices in operation in distribution networks have the expected smartness that allows for performing signifi- cant research on the smart grid functionalities. Therefore, this paper presents a methodology to develop a simulation platform for an energy management system in the smart grid environment, where all components of the DMS architecture are designed in accordance with the smart grid concepts (EPRI 2009; Cespedes 2012). Currently, some electrical system simulation platforms are being developed, for example, the platform developed by Sucic et al. (2011) requires proprietary software, such as DigSilent Power Factory and MatLab, which increases the project cost. Other platforms use a dedicated computer, limiting the researcher to a restricted physical area (Montenegro et al. 2012). In contrast, the proposed platform uses open-source software and mathematical models that can be compiled by any program language, which guarantees low cost and high efficiency. The proposed platform also supports Web-based simulations, where the researcher can experience and work with dynamic and interactive simulations (Granlund et al. 2000); as a result, the researcher is able to test hypotheses and perform experimentation and exploration to develop new smart process and products. In this way, the proposed platform contributes to the integration of the simulation, design, operation and analysis processes that represents a major challenge to the engineering of a smart grid system (zu Eissen and Stein 2006).

\section{Smart Grid Reference Models}

The challenges to deploy the smart grid are numerous, and many proposed solutions have been presented, creating a scenario of uncertainties and non-uniformity. To clarify the scenario, some study groups are developing smart grid standards. The International Telecommunication Union established the Focus Group on Smart Grid (FG-Smart), which developed the simplified reference smart grid architecture, as shown in Fig. 1, where the service provider, grid and customer domains are connected through a communication network that exchanges the information between them. The SCADA supervisory system at operation domain, for example, can control and collect data in real-time from the field devices in the grid domain via the communication network (Lee and Su 2013). The FG-Smart obtained the reference architecture by the division of the smart grid in three large areas: smart grid services/applications, communication and physical equipment that are linked to the layers 1, 2 and 3, respectively, in Fig. 1. This proposal of the smart grid architecture is layered similarly to the SCADA system design and the open system interconnection (OSI) model that also influenced the development of the complete reference model for the smart grid (Cespedes 2013).

Figure 2 presents the seven layers of the complete reference model for the smart grid: 
Fig. 1 Simplified smart grid reference architecture

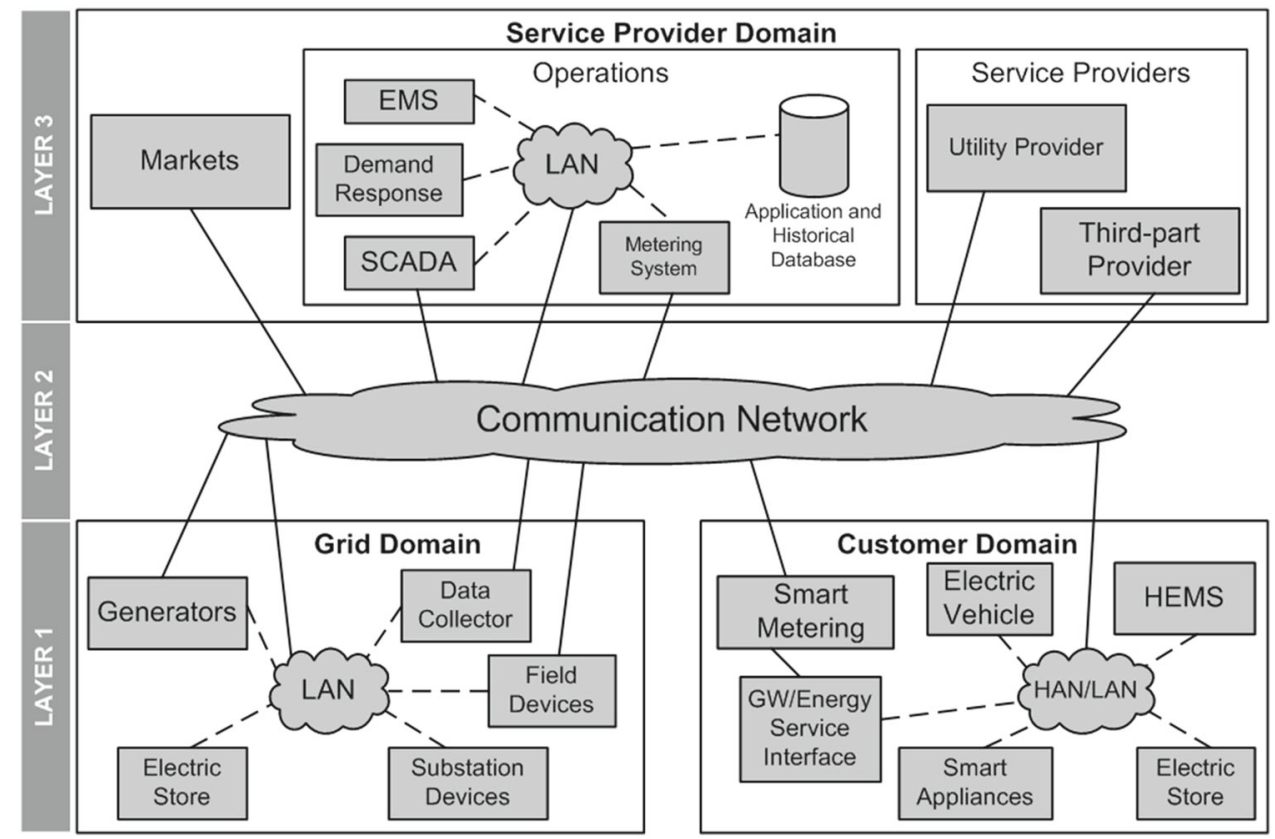

\begin{tabular}{|c|c|}
\hline I & Intelligence \\
\hline A & Analysis \\
\hline$M$ & Model \\
\hline S & System \\
\hline C & Communication \\
\hline If & Interface \\
\hline P & Physical \\
\hline
\end{tabular}

Fig. 2 Complete smart grid reference model

(1) Physical (P): includes all physical devices of the power system and equipment that provide the analog variables related to the operational state of the generators, lines, transformers, etc.;

(2) Interface (If): allows the connection and data transfer among the physical elements and the upper layers, and also includes all equipment that execute the analog-todigital conversion;

(3) Communication $(C)$ : includes all components that allow for the data exchange among the smart grid devices and supports several types of data transfer, for example, fiber optics, power line carrier and wireless;

(4) System $(S)$ : corresponds to the devices and applications of computational programs that collect and process the data from the communication, interface and physical layers;

(5) $\operatorname{Model}(M)$ : is an abstract mathematical model that aims to represent the elements from the system, communication, interface and physical layers;

(6) Analysis (A): is a set of computational programs that has all the functions and applications to support the operator decisions and uses the real-time data or historical data from the system layer;

(7) Intelligence (I): constitutes the highest layer and has the advanced data processing applications that eliminate or minimize human intervention;

The complete reference model for the smart grid is a high level conception that allows for differentiation of the smart grid projects from the traditional power system projects. Figure 3 shows a design example of an advanced DMS where all resources required by the tool of dynamic coloring are exposed.

In the physical layer, there is a distribution system where the medium- and low-voltage networks are connected by a distribution transformer that is monitored by the PMU \#1. Similarly, the circuit breaker is monitored and controlled by the intelligent electrical device (IED) \#1 and the automatic switch by the IED \#2, while the loads are monitored by the smart meters (SM) \#1 and \#2. All monitoring and controlling devices are located in the interface layer, where there are also the data aggregator units that transfer metering data via a wide-area network (WAN), represented by a cloud in the communication layer. The system layer primarily has the computational hardware, such as servers (market, geographical information system (GIS), database (DB), web) and clients (operator), that collect and process the data from the communication system. The four described layers have all the equipment of the electrical, communication and information systems. The three upper layers are logic layers and have computational programming applications that are the target of the many smart grid research studies, for example, the state estimation in the analysis layer is fundamental 
Fig. 3 The design of the advanced DMS using the complete reference model

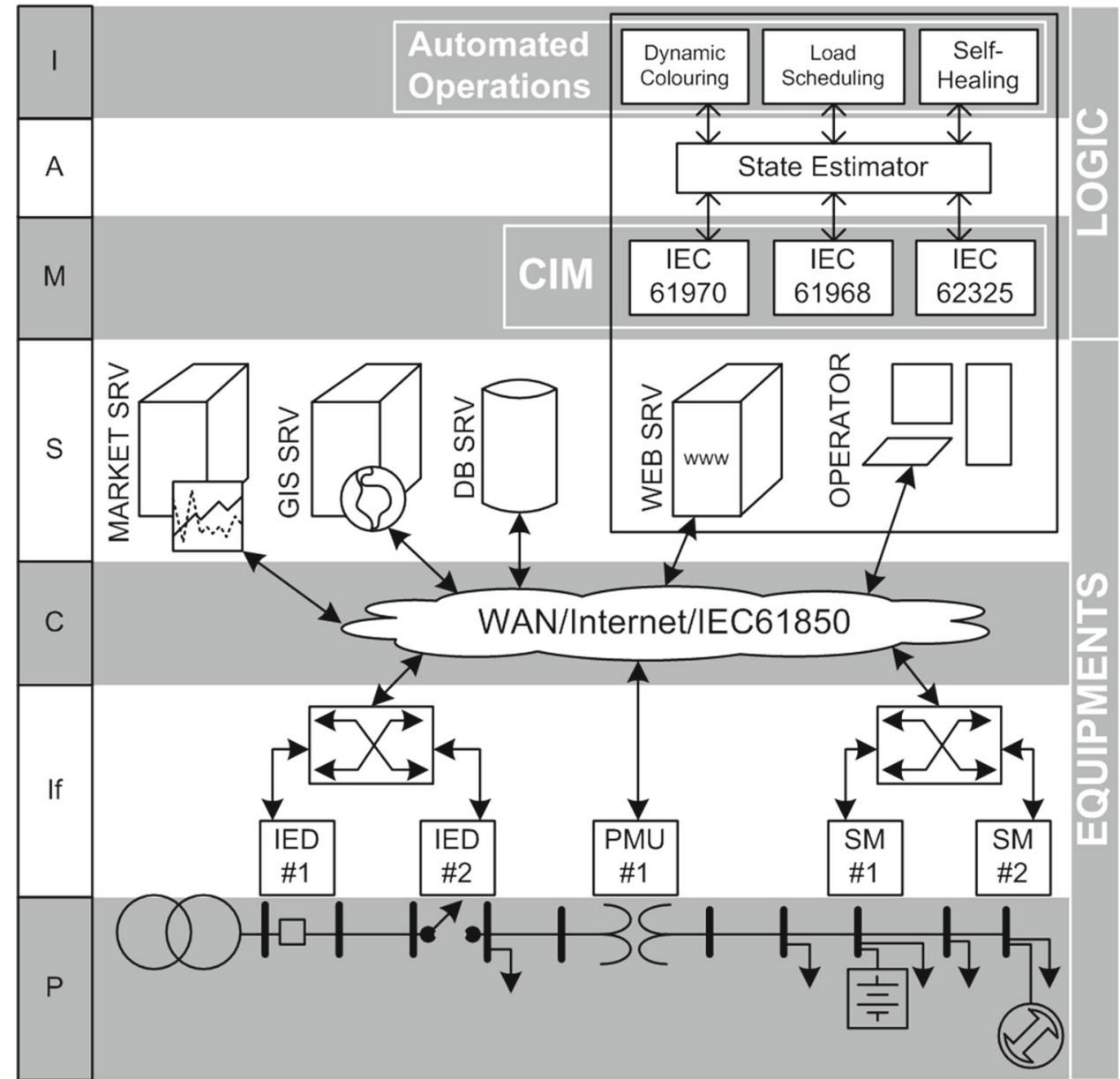

to operation of the advanced DMS and provides support to many of the automated operations of the intelligence layer, such as self-healing and load scheduling.

Because many research objectives are related to the three upper logic layers and the distribution networks currently in operation do not have the smart grid characteristics, the physical, interface and communication layers can be meticulously emulated using an electrical devices simulator.

\section{Formulation of the Physical Layer}

The power flow algorithms are an alternative way to simulate the behaviors of the electrical distribution networks in the physical layer.

\subsection{Deterministic Power Flow Algorithm}

The distribution network is composed by branches and busses; the branches are the series-connected electrical devices, such as lines and switches, and the busses are the shunting-connected electrical devices, such as loads, capacitors and generators. In this representation, the distribution transformer is also a series-connected device that connects the medium-voltage (MV) network to the low-voltage (LV) network and often causes a phase-angle deviation due to its connection type.

The mathematical representation of the distribution network is in the form of a graph tree, $G(v, u)$, where $v$ is a finite set of nodes that have the shunting-connected devices parameters, and $u$ is a finite set of edges that have the seriesconnected devices parameters. Thus, the deterministic power flow can be seen as a transference function, $H$.

$G\left(\dot{V}_{i}, \dot{J}_{i}\right)=\left.H\left(G\left(\dot{S}_{i}, M_{i}\right)\right)\right|_{\dot{V}_{0}=\dot{V} O P}, \quad i=0,1,2, \ldots, m$

In the deterministic power flow algorithm, first, the nodes and edges are arranged through the distribution network topology, where the elements that are more peripheral have a major index, $i$, than the elements closer to the substation, so the most peripheral element has the index equal to $m$. Moreover, the algorithm input data are the power apparent injections, $\dot{S}_{i}$, of the shunting-connected devices and the edge matrix models, $M_{i}$, of the series-connected devices. After the 


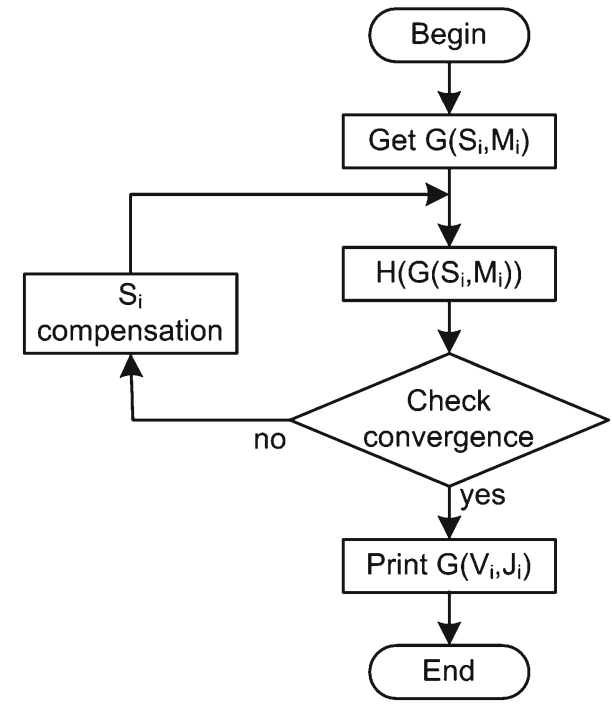

Fig. 4 Flowchart of the complete deterministic power flow

power flow calculation, the nodes have bus voltage values, $\dot{V}_{i}$, and the edges have branch current values, $\dot{J}_{i}$, in the output graph. If an algorithm of the backward/forward sweep is used, then the voltage of the root node is equal to the operating voltage of distribution network, $V^{O P}$, (Cheng and Shirmohammadi 1995).

$$
\begin{aligned}
{\left[\begin{array}{c}
{\left[\dot{J}_{i}\right]_{3 \times 1}} \\
{\left[\dot{V}_{i}\right]_{3 \times 1}}
\end{array}\right]=} & {\left[\begin{array}{l}
{\left[A_{i}\right]_{3 \times 3}\left[\bar{B}_{i}\right]_{3 \times 3}} \\
{\left[\bar{C}_{i}\right]_{3 \times 3}\left[D_{i}\right]_{3 \times 3}}
\end{array}\right] } \\
& \times\left[\begin{array}{c}
\sum\left[\dot{J}_{d}\right]_{3 \times 1}+\left[\left(\frac{\dot{S}_{i}}{\dot{V}_{i}}\right)^{*}\right]_{3 \times 1} \\
{\left[\dot{V}_{u}\right]_{3 \times 1}}
\end{array}\right] .
\end{aligned}
$$

Equation (2) is a compact representation of $H$ with all the input and output variables for the three-phase deterministic power flow calculation. The edge matrix model, $\left[M_{i}\right]_{6 \times 6}$, corresponds to a matrices set where the submatrices $\left[A_{i}\right]_{3 \times 3}$ and $\left[D_{i}\right]_{3 \times 3}$ are dimensionless matrices, $\left[\bar{B}_{i}\right]_{3 \times 3}$ is an admittance matrix, and $\left[\bar{C}_{i}\right]_{3 \times 3}$ is an impedance matrix. In the procedure of the backward sweep, the algorithm calculates the branch currents, $\dot{J}_{i}$, using the known values of the adjacent downstream currents that are tagged by the index $d$, and, in the forward sweep, the algorithm calculates bus voltages, $\dot{V}_{i}$, using the known values of adjacent upstream voltages that are tagged by the index $u$.

The previous power flow formulation solves a radial distribution network without any voltage controlled busses. The algorithm can also use a power compensation routine when there is a weakly meshed distribution network or a distributed generator. Figure 4 shows the complete deterministic power flow with a $S_{i}$ compensation routine that computes power injection corrections in a bus $i$ until the convergence is reached.
The different electrical devices are formulated according to connection type, as presented below.

\section{A Series Connected Devices}

(1) Distribution Line: the feeders of the distribution network have either overhead or underground three-phase branches modeled through their series impedances, $\bar{Z}_{l_{i}}$.

$$
\left[\begin{array}{l}
{\left[A_{i}\right]_{3 \times 3}\left[\bar{B}_{i}\right]_{3 \times 3}} \\
{\left[\bar{C}_{i}\right]_{3 \times 3}\left[D_{i}\right]_{3 \times 3}}
\end{array}\right]=\left[\begin{array}{ll}
{[I]_{3 \times 3}} & {[\overline{0}]_{3 \times 3}} \\
-\left[\bar{Z}_{l_{i}}\right]_{3 \times 3}[I]_{3 \times 3}
\end{array}\right] .
$$

The distribution line model was adapted from (Alhajri et al. 2010), where $[I]_{3 \times 3}$ is the identity matrix, $[\overline{0}]_{3 \times 3}$ is the zero matrix and $\left[\bar{Z}_{l_{i}}\right]_{3 \times 3}$ is the three-phase impedance matrix.

(2) Distribution Transformer: is an electrical device very common in the distribution network and converts MV to LV.

$$
\left[\begin{array}{l}
{\left[A_{i}\right]_{3 \times 3}\left[\bar{B}_{i}\right]_{3 \times 3}} \\
{\left[\bar{C}_{i}\right]_{3 \times 3}\left[D_{i}\right]_{3 \times 3}}
\end{array}\right]=\left[\begin{array}{ll}
K_{n}^{-1}\left[\Phi_{i}\right]_{3 \times 3} & {\left[\bar{Y}_{m i}\right]_{3 \times 3}} \\
-\left[\bar{Z}_{D i}\right]_{3 \times 3} & K_{n}^{-1}\left[\Phi_{i}\right]_{3 \times 3}^{\mathrm{T}}
\end{array}\right] .
$$

The distribution transformer parameters come from the information card and from the short- and open-circuit tests. In (4), $\left[\overline{Y_{m i}}\right]_{3 \times 3}$ and $\left[\overline{Z_{D i}}\right]_{3 \times 3}$ are diagonal matrices are filled by the magnetization admittance and the dispersion impedance, respectively, $K_{n}$ is the transformation ratio, and $\left[\Phi_{i}\right]_{3 \times 3}$ is the matrix of phase-angle deviation resultant of the incidence analysis at the transformer terminals for the different types of transformer connections.

\section{B Shunting Connected Devices}

(1) Capacitor: the bank of capacitors is used to compensate the power factor and can reduce the line losses along the distribution network through the canceling of the reactive power of the motors or any other low power factor devices (Grainger and Civanlar 1985). The bank of capacitors can also regulate the network voltage when its capacitors are switched, as formulated by (5).

$\dot{S}_{i}^{p}(s)=0-j \frac{s Q^{N O M}}{3 s^{M A X}}, \quad p=a, b, c$.

The specification of the bank capacitors corresponds to its total three-phase reactive power, $Q^{N O M}$; thus, each phase, $p$, has one-third of the total power. Furthermore, a switched bank of capacitors depends on the step, $s$, which controls the amount of reactive power injected to 
the distribution network. In this way, $s$ must be a positive integer that can reach a maximum value equal to $s^{M A X}$.

(2) Distributed Generator: an electrical device that is becoming more common within the distribution system and can reduce losses, enhance the voltage profile and increase the reliability. The distributed generator is modeled as either a PQ or PV bus (Khushalani et al. 2007). If the PV bus model is adopted, the voltage in the generator bus, $\left|\dot{V}_{i}^{p}\right|$, is compared to a previously specified voltage, $\left|\dot{V}_{S P}^{p}\right|$, for all iterations until convergence occurs; in other words, until the voltage difference, calculated by (6), becomes less than a maximum tolerable error of voltage, $\varepsilon_{\mathrm{GD}}$, as given by (7).

$$
\begin{aligned}
& \Delta V_{i}^{p}=\left|\dot{V}_{S P}^{p}\right|-\left|\dot{V}_{i}^{p}\right| \\
& \max _{p=a, b, c}\left\{\left|\Delta V_{i}^{p}\right|\right\} \leq \varepsilon_{D G}
\end{aligned}
$$

Because the voltage depends strongly on the generator reactive power, the power compensation routine adjusts the reactive power of the distributed generator, $Q_{D G}^{p}$, between a maximum reactive power, $Q_{D G}^{\max }$, and a minimal reactive power, $Q_{D G}^{m i n}$, to decrease the voltage difference, $\Delta V_{i}^{p}$. The power compensation method is given by (8), where the reactive power required to eliminate the voltage difference is added to the reactive power of the anterior iteration, $Q_{D G \text {,anterior }}^{p}$.

$Q_{D G}^{p}=i m g\left\{\dot{V}_{i}^{p}\left(\Delta \dot{I}_{i}^{p}\right)^{*}\right\}+Q_{D G, \text { anterior }}^{p}$

Equation (9) states that the current injection, $\Delta \dot{V}_{i}^{p}$, used to calculate the reactive power correction, depends on the voltage difference and the serial short-circuit impedance, $\bar{Z}_{S C}$, between the distributed generator and the source bus.

$\Delta \dot{I}_{i}^{p}=\frac{\left|\Delta V_{i}^{p}\right|}{\left|\bar{Z}_{S C}\right|} e^{j\left(\operatorname{sgn}\left(\Delta V_{i}^{p}\right) \frac{\pi}{2}+\operatorname{ang}\left(\dot{V}_{i}^{p}\right)\right)}$.

If the reactive power violates the specified limits during the power compensation routine, then the reactive power of the distributed generator is fixed at a limiting value $\left(Q_{D G}^{\max }\right.$ or $\left.Q_{D G}^{m i n}\right)$ and its bus is now treated as a PQ bus model.

(3) Load: the end consumer connection point that is modeled by the consumed apparent power and the mixed behavior resulting from the connection of different electrical appliances.

$S_{i}^{p}=\frac{S_{i}^{N O M}}{3} \sum_{q=0}^{2}\left[\lambda_{q}\left(\frac{\left|\dot{V}_{i}^{p}\right|}{\left|\dot{V}_{i}^{N O M}\right|}\right)^{q}\right]$.

In (10), $S_{i}^{N O M}$ is the magnitude of the three-phase nominal apparent power, and $V_{i}^{N O M}$ is the three-phase nominal

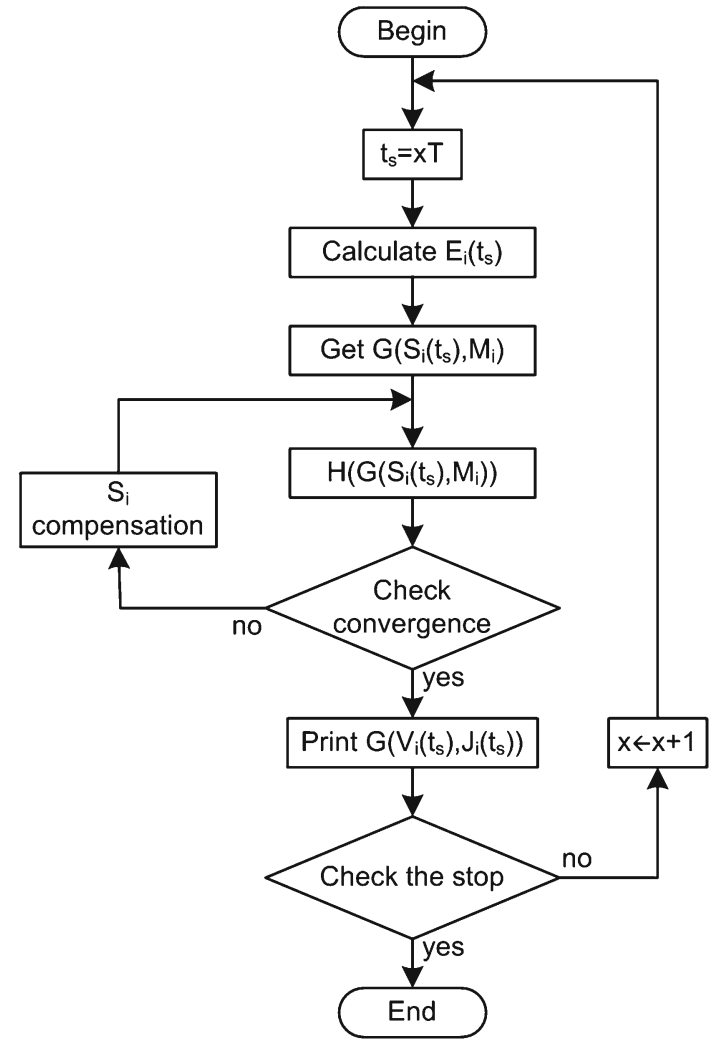

Fig. 5 Flowchart of the probabilistic power flow

voltage of the bus $i$. The index $q$ indicates the load behavior: $q=0$ (constant power), $q=1$ (constant current), and $q=2$ (constant impedance). Moreover, the load can simultaneously have the three behaviors weighted by a factor of value $\lambda_{q}$, whose sum is equal to one. This load model, also known as the ZIP model, is deterministic and fixed over time, thus, the multiplication of the value of $S_{i}^{p}$ by a time function produces a load model, where the load power value, $S_{i}^{p}(t s)$, is closer to the real behavior.

$\dot{S}_{i}^{p}\left(t_{s}\right)=S_{i}^{p} \dot{E}_{i}^{p}\left(t_{s}\right)$

The time function, $\dot{E}_{i}^{p}\left(t_{s}\right)$, is the percentage of energy consumption over time and has a non-deterministic feature that is required by the probabilistic power flow algorithm.

\subsection{Probabilistic Power Flow Algorithm}

The percentage of energy consumption is expressed either as the ratio between the installed and consumed power or as the power demand over time that is measured hourly. The proposed methodology uses a discrete time interval, $T$, which is the simulated metering interval and can be adjusted by the operator during the performance of the probabilistic power flow algorithm, Fig. 5. 


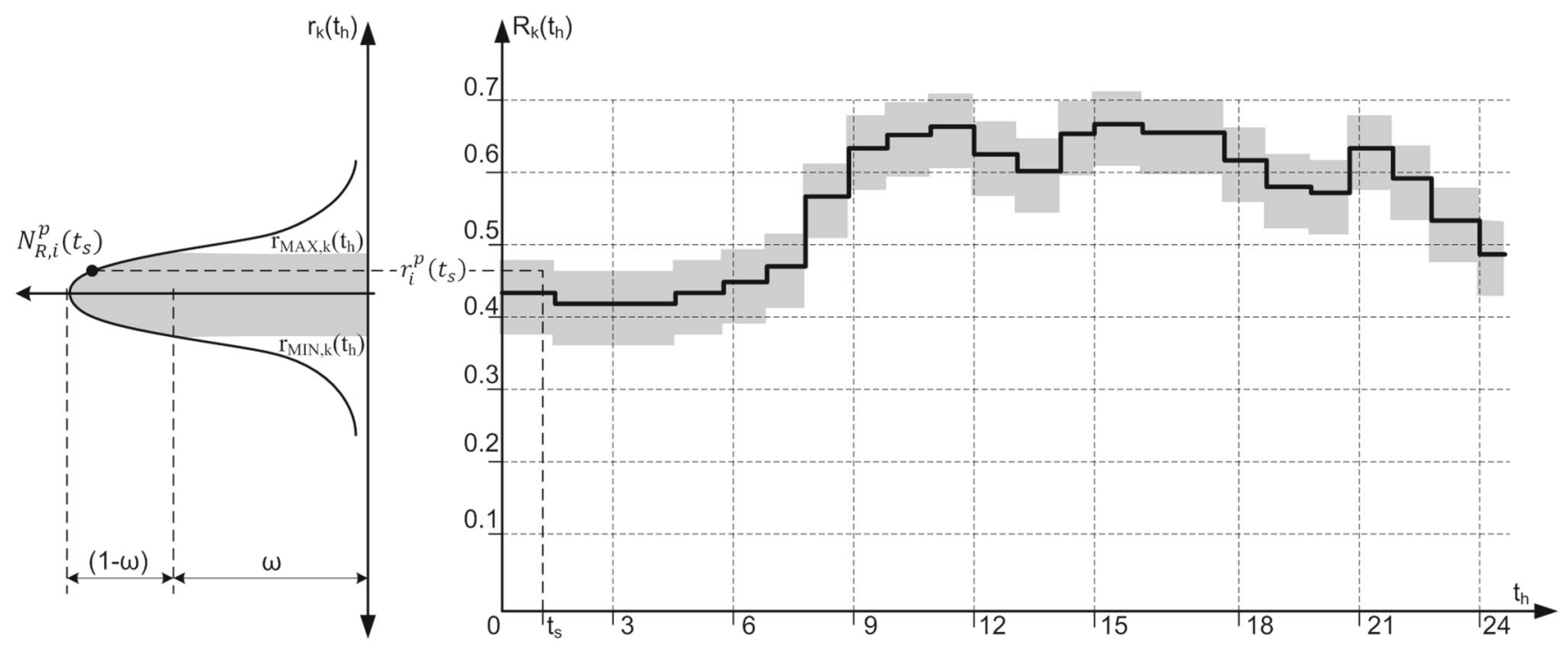

Fig. 6 Fluctuation range of the power magnitude over time

The electrical devices simulator reproduces the distribution system behavior using the probabilistic power flow algorithm that generates the current and voltage values until the operator stops it. In each iteration, $x$, the algorithm increases one $T$ in $t_{s}$, calculates the percentage of the energy consumption and performs the computation of a deterministic power flow; in this way, the load fluctuates over time continuously.

\section{A Function of the Percentage of Energy Consumption}

The function of the percentage of energy consumption is a complex number because it represents the power demand of the load that is just specified by the magnitude of the apparent power.

$\dot{E}_{i}^{p}\left(t_{s}\right)=r_{i}^{p}\left(t_{s}\right) e^{j \cos ^{-1} f_{i}^{p}\left(t_{s}\right)}$.

The monitoring of the traditional existing distribution systems is performed at the substation, where the hourly measurements of the normalized magnitude, $R\left(t_{h}\right)$, and power factor, $F\left(t_{h}\right)$, of the power consumed for each phase are collected. The proposed methodology calculates the normalized magnitude, $r_{i}^{p}\left(t_{h}\right)$, and power factor, $f_{i}^{p}\left(t_{h}\right)$, of the power consumed by each load using a normal probability density function, $N(y)$.

$N(y)=\frac{1}{\sigma \sqrt{2 \pi}} e^{-\frac{(y-\mu)^{2}}{2 \sigma^{2}}}$.

In (13), the random variable is $y$, while $\mu$ and $\sigma$ are the mean and standard deviation, respectively, of the variable $y$, which can have the values of either $R\left(t_{h}\right)$ or $F\left(t_{h}\right)$. If $y\left(t_{h}\right)$ is an hourly function with a specific behavior for each hour, $t_{h}$, then the variance of hourly normalized magnitude, $\sigma_{R}\left(t_{h}\right)^{2}$, and the variance of hourly power factor, $\sigma_{F}\left(t_{h}\right)^{2}$, can be expressed by (14) and (15), respectively.

$\sigma_{R}\left(t_{h}\right)^{2}=\frac{\sum_{k=1}^{n}\left(R_{k}\left(t_{h}\right)\right)^{2}-\frac{\left(\sum_{k=1}^{n} R_{k}\left(t_{h}\right)\right)^{2}}{n}}{n-1}$

$\sigma_{F}\left(t_{h}\right)^{2}=\frac{\sum_{k=1}^{n}\left(F_{k}\left(t_{h}\right)\right)^{2}-\frac{\left(\sum_{k=1}^{n} F_{k}\left(t_{h}\right)\right)^{2}}{n}}{n-1}$.

In the variance calculation, $n$ is the total amount of metering days and $k$ is the metering day. As the probability density function strongly depends on the calculated variance, a point at the normal curve can be easily chosen.

$N_{R, i}^{p}\left(t_{s}\right)=\frac{\left(\omega+\alpha_{R, i}^{p}\left(t_{s}\right)(1-\omega)\right)}{\sigma_{R}\left(t_{h}\right) \sqrt{2 \pi}}$
$N_{F, i}^{p}\left(t_{s}\right)=\frac{\left(\omega+\alpha_{F, i}^{p}\left(t_{s}\right)(1-\omega)\right)}{\sigma_{F}\left(t_{h}\right) \sqrt{2 \pi}}$.

In (16) and (17), $\alpha_{R, i}^{p}\left(t_{s}\right)$ and $\alpha_{F, i}^{p}\left(t_{s}\right)$ are the random real variables, from 0 to 1 , and the projections of the points $N_{R, i}^{p}\left(t_{s}\right)$ and $N_{F, i}^{p}\left(t_{s}\right)$ over the abscissa indicate the values of $r_{i}^{p}\left(t_{s}\right)$ and $f_{i}^{p}\left(t_{s}\right)$, respectively. Hence, the variable $\omega$ limits the fluctuation range between the maximum and minimum projection. For example, Fig. 6 shows a normal curve, where $\omega$ limits the value of $r_{i}^{p}\left(t_{s}\right)$ between $r_{M A X, k}\left(t_{h}\right)$ and $r_{M I N, k}\left(t_{h}\right)$.

$$
\begin{aligned}
& r_{i}^{p}\left(t_{s}\right)= \pm \sqrt{2 \sigma_{R}\left(t_{h}\right)^{2} \ln \left(\frac{1}{N_{R, i}^{p}\left(t_{s}\right) \sigma_{R}\left(t_{h}\right) \sqrt{2 \pi}}\right)}+R_{k}\left(t_{h}\right) \\
& f_{i}^{p}\left(t_{s}\right)= \pm \sqrt{2 \sigma_{F}\left(t_{h}\right)^{2} \ln \left(\frac{1}{N_{F, i}^{p}\left(t_{s}\right) \sigma_{F}\left(t_{h}\right) \sqrt{2 \pi}}\right)}+F_{k}\left(t_{h}\right) .
\end{aligned}
$$


In (18), the operator \pm before the square root symbol can be replaced by $\operatorname{sgn}(\beta-1 / 2)$, where $\beta$ is a random real variable, from 0 to 1 , and $\operatorname{sgn}($.) is the signal function that returns the value of -1 in the case that $\beta-1 / 2$ is negative or the value of 1 in the case that $\beta-1 / 2$ is positive. This equation is the inverse to the normal distribution, where the mean is replaced by the hourly measurement of the normalized magnitude, $R_{k}\left(t_{h}\right)$. For example, Fig. 6 shows the behavior of the normalized power magnitude over time, where the value of $r_{i}^{p}\left(t_{s}\right)$ is randomly calculated for each time interval and whose the result is inside of the fluctuation range that surrounds the measurement $R_{k}\left(t_{h}\right)$; thereby, a continuous magnitude variation is reproduced by the electrical devices simulator. In the same way, the simulator reproduces the power factor variation by (19).

Until now, issues involving the time of the algorithm were not clearly explained; one of these issues is the relationship between the simulation time, $t_{s}$, and the measurement time, $t_{h}$. In (20), the value of $t_{h}$ is the hour of the day when one measurement is taken; the floor operator, L.J, always returns the largest previous integer, and the value of $t_{s}$ is the simulation time in minutes. As $t_{s}$ is increased by step $T$, if $T=15$ min, for example, then the algorithm produces four simulated measurements for each taken measurement.

$t_{h}=\left\lfloor\frac{t_{s}}{60}\right\rfloor-24 k$

Similar to the measurement time, the variable $k$ represents the day when the measurement is taken and is calculated by: $k=t_{s} / 1440$.

\section{B Consumption Influenced by the Market}

There is a substantial amount of information that flows between the electrical, monitoring and controlling devices in the modern distribution system. Thus, the end consumer can have a home energy management system (HEMS) that receives information from the market and sends information to the advanced DMS.

Table 1 outlines the typical distributed energy resource (DER) units, which include the distributed generation (DG) and storage (DS) units, and the possibility of power flow control; thereby, the end consumer can have a combination of DG, DS and consumption units, as shown in Fig. 7, that are controlled and monitored by the HEMS; consequently, during the periods of high energy cost, the generation unit is activated, reducing the power demand consumed from the distribution network and, during the periods when the energy costs are low, the storing and consumption units are activated, increasing the power demand consumed from the distribution network. That operation becomes possible due to the exchange of information of the energy cost variation between
Table 1 Typical DER units into the smart grid (Katiraei et al. 2008)

\begin{tabular}{lll}
\hline Device type & $\begin{array}{l}\text { Primary energy } \\
\text { source }\end{array}$ & $\begin{array}{l}\text { Power flow } \\
\text { control }\end{array}$ \\
\hline Conventional DG & $\begin{array}{l}\text { Reciprocating } \\
\text { engines small } \\
\text { hydro } \\
\end{array}$ & $+\mathrm{P}, \pm \mathrm{Q}$ \\
& $\begin{array}{l}\text { Fixed-speed wind } \\
\text { turbine }\end{array}$ & $+\mathrm{P},-\mathrm{Q}$ \\
Nonconventional DG & $\begin{array}{l}\text { Variable-speed wind } \\
\text { turbine }\end{array}$ & $+\mathrm{P}, \pm \mathrm{Q}$ \\
& $\begin{array}{l}\text { Microtubine } \\
\text { Solar PV }\end{array}$ & $+\mathrm{P}, \pm \mathrm{Q}$ \\
& Fuel cell & $+\mathrm{P}, \pm \mathrm{Q}$ \\
Long-term DS & Battery storage & $\pm \mathrm{P}, \pm \mathrm{Q}$ \\
Short-term DS & Super capacitor & $\pm \mathrm{P}, \pm \mathrm{Q}$ \\
& Flywheel & $\pm \mathrm{P}, \pm \mathrm{Q}$ \\
\hline
\end{tabular}

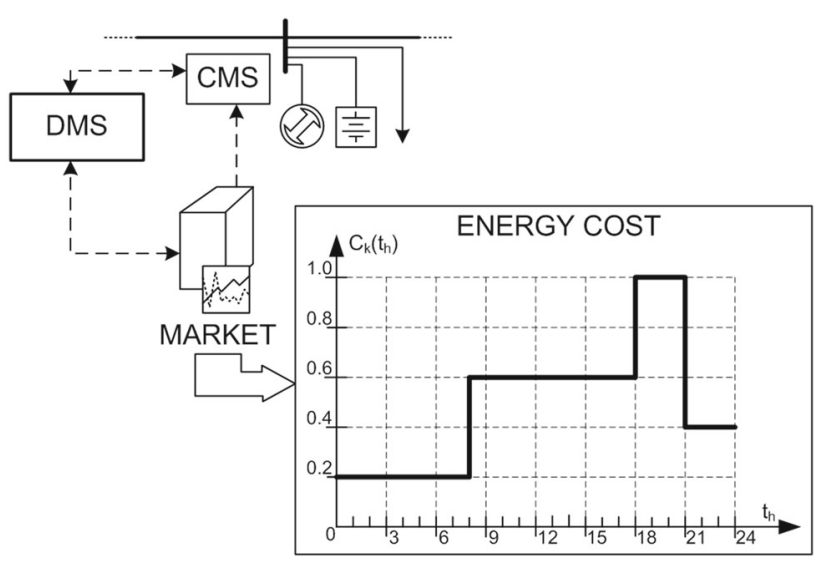

Fig. 7 Diagram of the communication structure and energy cost fluctuation

the energy market and the HEMS; hence, the percentage of energy consumption should be a function of energy cost, $C_{k}\left(t_{h}\right)$.

Equations (21) and (22) express an alternative way to calculate the normalized power magnitude and power factor of the consumption influenced by the market, respectively.

$r_{i}^{p}\left(t_{s}\right)=r_{0}-\delta_{R} C_{k}\left(t_{h}\right)$

$f_{i}^{p}\left(t_{s}\right)=f_{0}+\delta_{F} C_{k}\left(t_{h}\right)$.

Both equations are linear functions of the HEMS power controller. In (21), $r_{0}$ is the consumer's total installed power, and $\delta_{R}$ is a conversion coefficient that is calculated by the mean of ratio between the amount of consumed power and the energy cost. Similarly, in (22), $f_{0}$ is the minimum power factor value acceptable in the distribution network, and $\delta_{F}$ is a conversion coefficient that makes the load power factor equals to one when the energy cost is maximal because the power demand from the distribution network must be 
minimal according to (21). Equations (21) and (22) are used as controllers of consumed power; they are also examples of the application of the proposed method of probabilistic power flow.

\section{Formulation and Virtualization of the Interface Layer}

The interface layer has several types of electrical sensors located along the distribution network that convert analog measurements to digital values. In this way, the emulations of the IEDs, PMUs and SMs are performed by the addition of the quantization error into the monitored electrical parameters and by the virtualization of the data exchange structure.

\subsection{Quantization Error}

The probabilistic power flow algorithm uses a doubleprecision floating-point to calculate the electrical parameters, such as voltage and current; thus its accuracy is of 53 bits, approximately 16 decimal digits. However, the devices of interface layer have analog-to-digital converters (ADC) of minor precision, for example, a smart meter typically has an accuracy of 12 bits (STMicroelectronics 2012), while the resolution of modern IED and PMU usually is 16 bits (Dutta and Guan 2008). The difference of accuracy causes the quantization error that is a form of noise of uniform distribution introduced by the ADC and can be simulated through the rounding of the magnitudes of the voltage and current.

$$
\begin{aligned}
& \left|\dot{V}_{A D C, i}^{p}\left(t_{s}\right)\right|=2^{(1-b)} V_{\text {ref }}\left\lfloor\frac{\left|\dot{V}_{i}^{p}\left(t_{s}\right)\right|}{2^{(1-b)} V_{\text {ref }}}+\frac{1}{2}\right\rfloor \\
& \left|\dot{I}_{A D C, i}^{p}\left(t_{s}\right)\right|=2^{(1-b)} I_{\text {ref }}\left\lfloor\frac{\left|\dot{I}_{i}^{p}\left(t_{s}\right)\right|}{2^{(1-b)} I_{\text {ref }}}+\frac{1}{2}\right\rfloor .
\end{aligned}
$$

In (23), $V_{\text {ref }}$ is the reference voltage of the electrical network and $\left|\dot{V}_{A D C, i}^{p}\left(t_{s}\right)\right|$ is the rounded value of the voltage magnitude that simulates the output signal of an ADC with $b$ bits of precision. In the same way, Eq. (24) expresses the rounded value of the current magnitude.

The quantization error only affects the magnitude values because the frequency of electrical signal is low; consequently, the phase distortion caused by the ADC is insignificant.

\subsection{Gateway Network Simulation}

The probabilistic power flow algorithm calculates the voltage and current values for the entire distribution network; however, in the real world, the management system only knows the set of electrical parameters, $\psi_{i}\left(t_{s}\right)$, metered by the monitoring units, as given by (25). Thus, the emulation of the

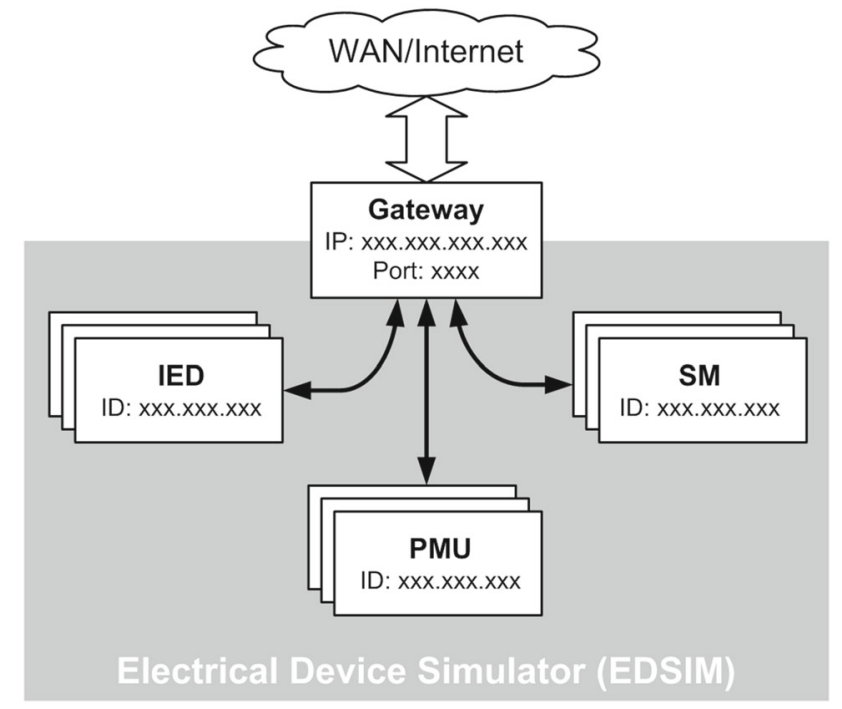

Fig. 8 Access structure to the monitoring units

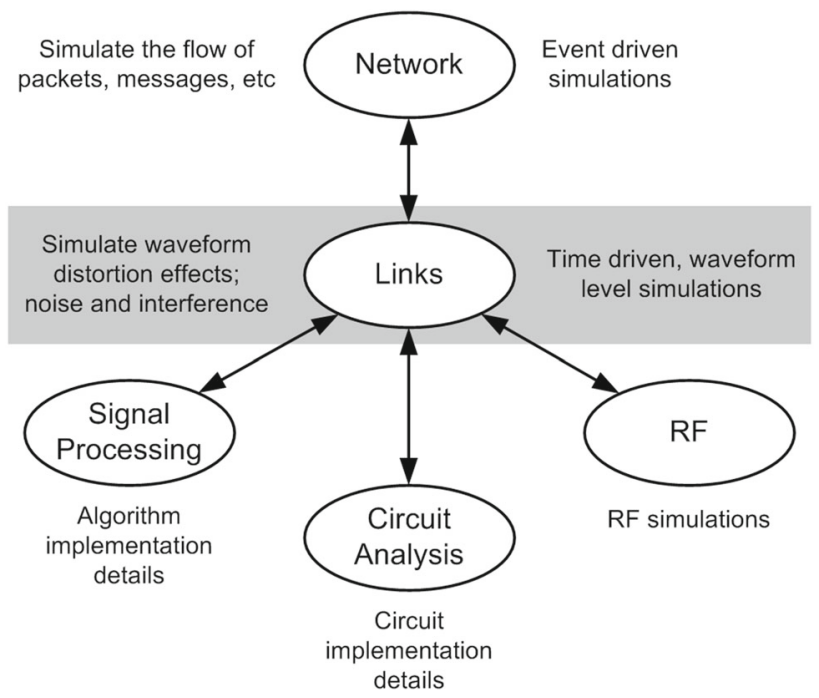

Fig. 9 Links level in the hierarchical view of communication systems

interface layer also requires the individualization of monitoring units.

$\psi_{i}\left(t_{s}\right)= \begin{cases}\dot{V}_{A D C, i}^{p}\left(t_{s}\right) \cup \dot{I}_{A D C, i}^{p}\left(t_{s}\right) & \text { if } i \text { th bus has SM } \\ \dot{V}_{A D C, i}^{p}\left(t_{s}\right) \cup \dot{J}_{A D C, i}^{p}\left(t_{s}\right) & \text { if } i \text { th bus has PMU } \\ \dot{V}_{A D C, i}^{p}\left(t_{s}\right) \cup \dot{J}_{A D C, i}^{p}\left(t_{s}\right) & \text { if } i \text { th bus has IED } \\ \emptyset & \text { otherwise }\end{cases}$

A feasible solution is obtained by the use of any object oriented programming language, where the monitoring units can be built as objects and following the design specifications. In addition, the information exchange with external world can be performed through a gateway, as shown in Fig. 8. 


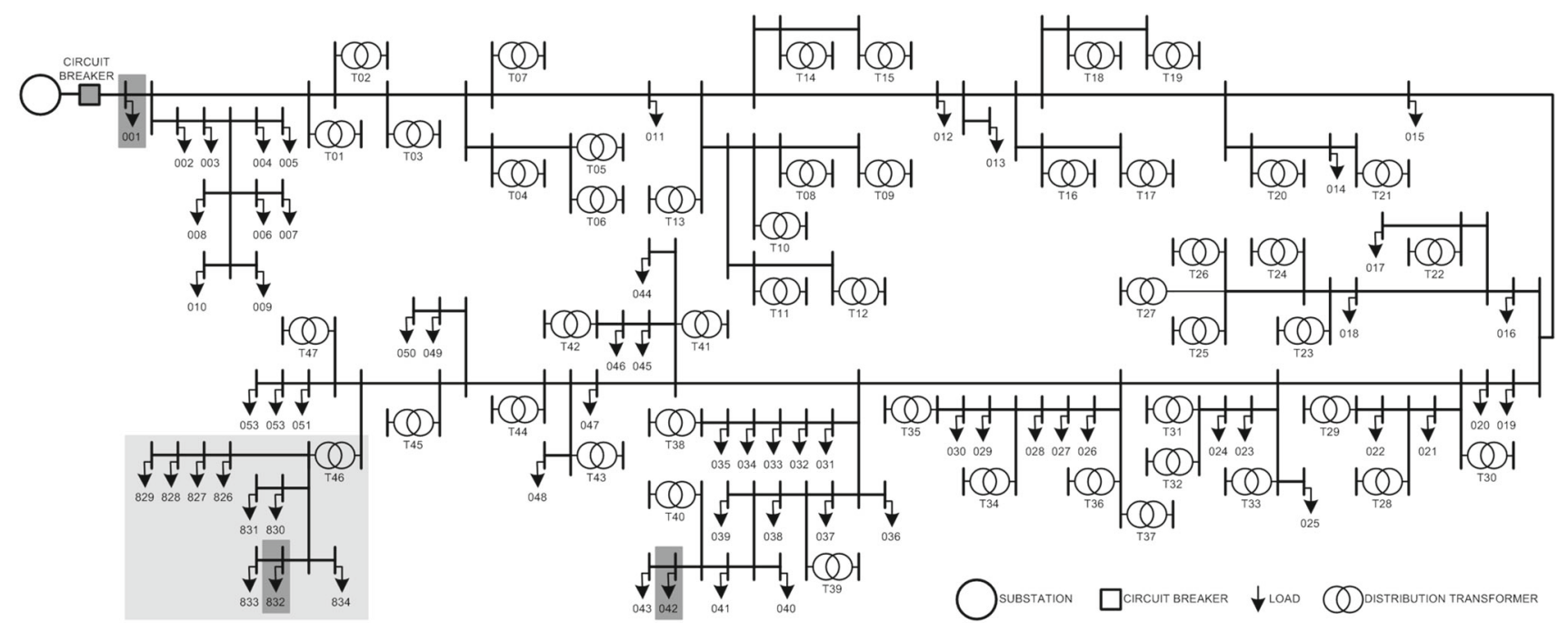

Fig. 10 Topologic diagram of a real distribution network

\section{Voltage Profile}
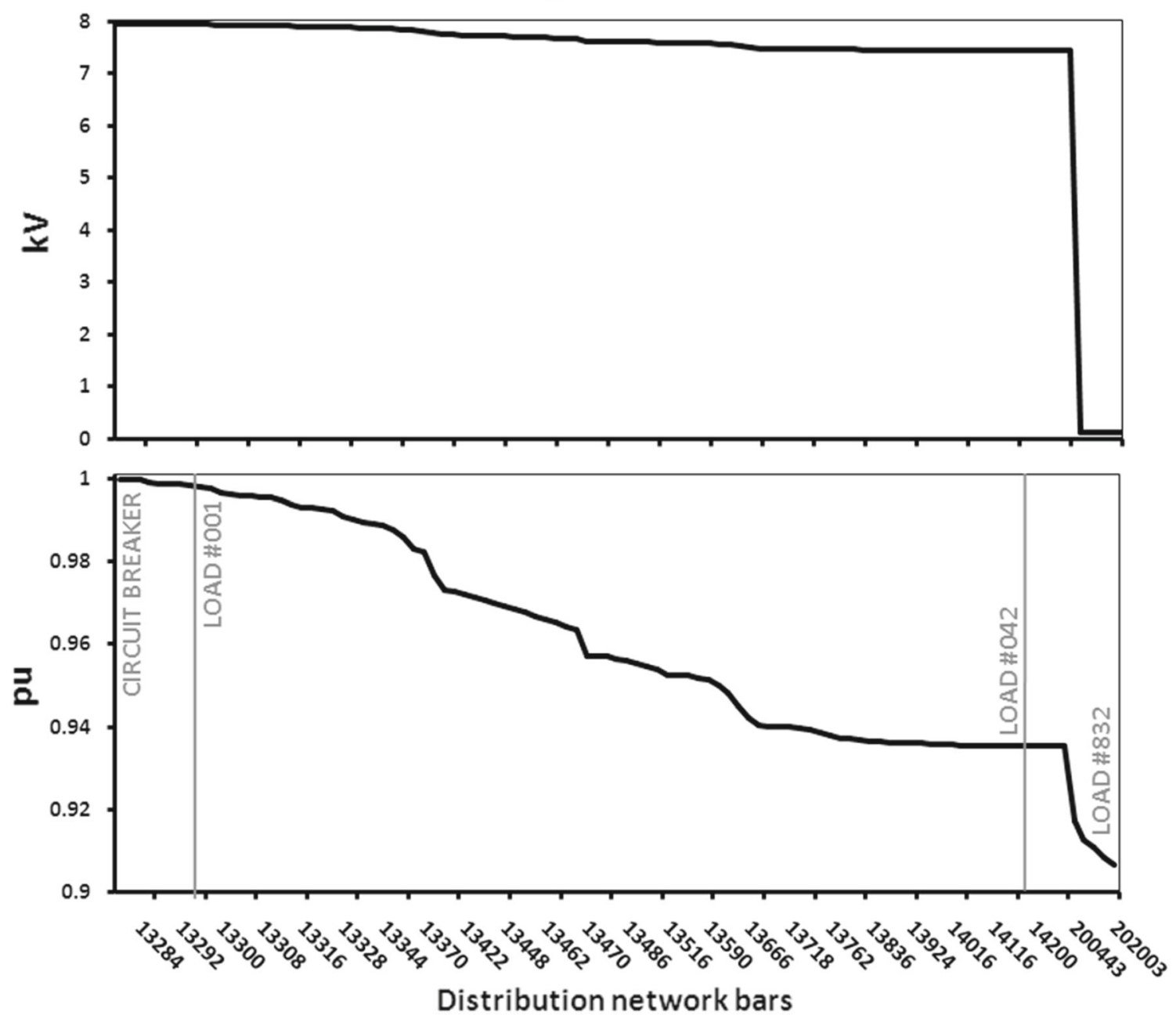

Fig. 11 Voltage profile from the substation to bus \#832 


\section{Cumulative frequency of demand}
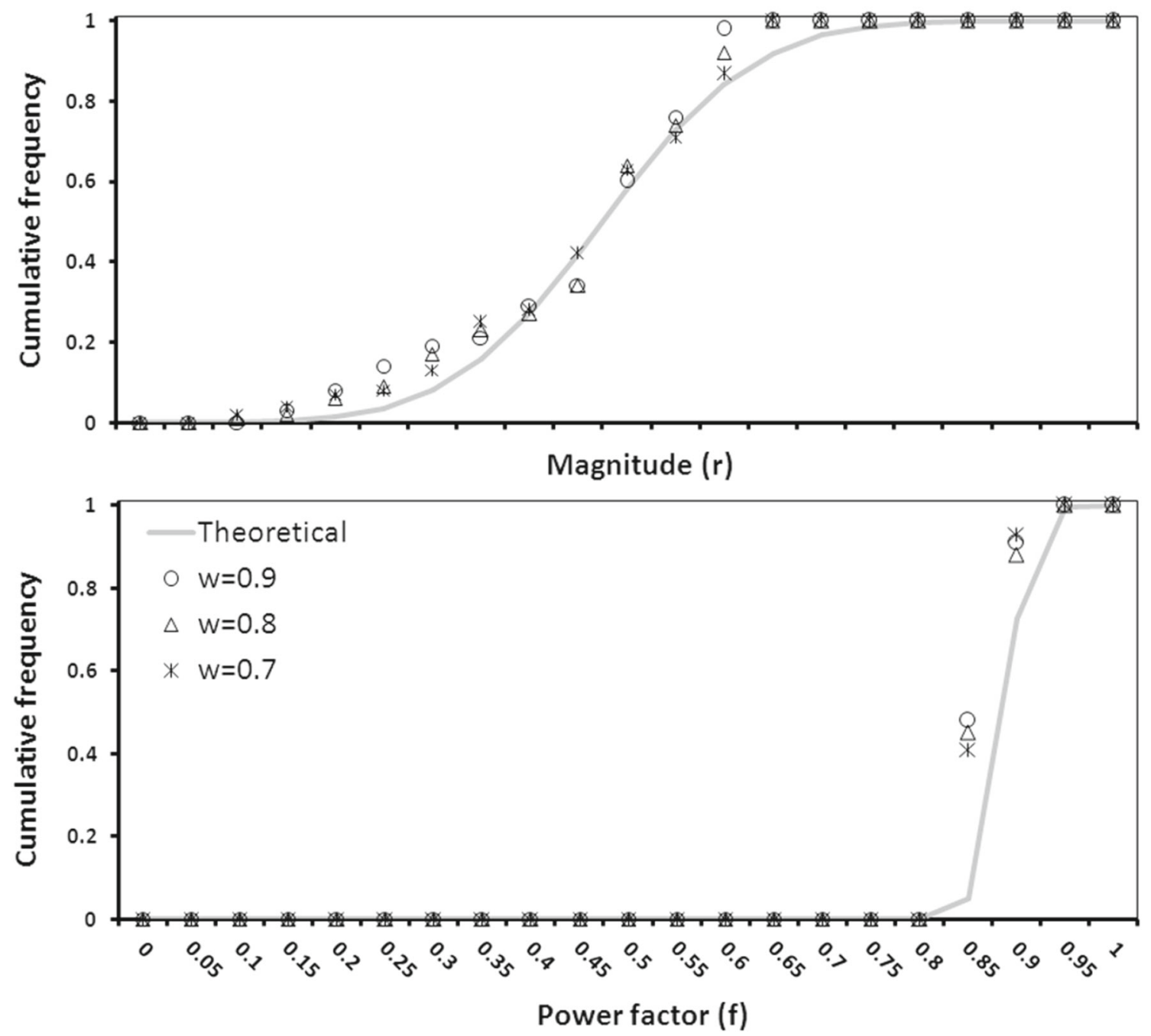

Fig. 12 Cumulative frequency curve of the load \#001 at noon

The management system connects to the electrical devices simulator (EDSIM) through the number of the IP address and of an opened access port. After the connection confirmation, one identification number (ID) is required to connect to the monitoring unit and, thus, insert or collect data.

\section{Emulation of the Communication Layer}

Communication systems can be evaluated through simulation-based approaches, where systems can be modeled with almost any level of desired detail, and the design space can be explored more finely (Jeruchim et al. 2002). A variety of simulation techniques are used to evaluate the performance of the different levels of the communication system that can be represented by a hierarchical view, as shown in Fig. 9. At the network level, the flow of packets and messages over the network is simulated using an event-driven simulator. In the middle level, the communication links address the transmission of information over different types of communication channels (free space, cables, wires, optical fibers, etc.), and the performance of the communication links is measured in terms of the bit error characteristics. The communication link is above components such as analog circuits, digital circuits, or algorithms implemented on a programmable digital processor that performs the signal processing operations.

The smart grid interoperability can be achieved through the standard IEC 61850 that allows for the free exchange of information among the different equipment independent from the communication network (Shawkat Ali 2013). In addition, the communication networks are assumed free of the congestion failures; hence, the target of the emulation is the communication channel that is the medium used to transmit the information and may be perturbed by the noise 

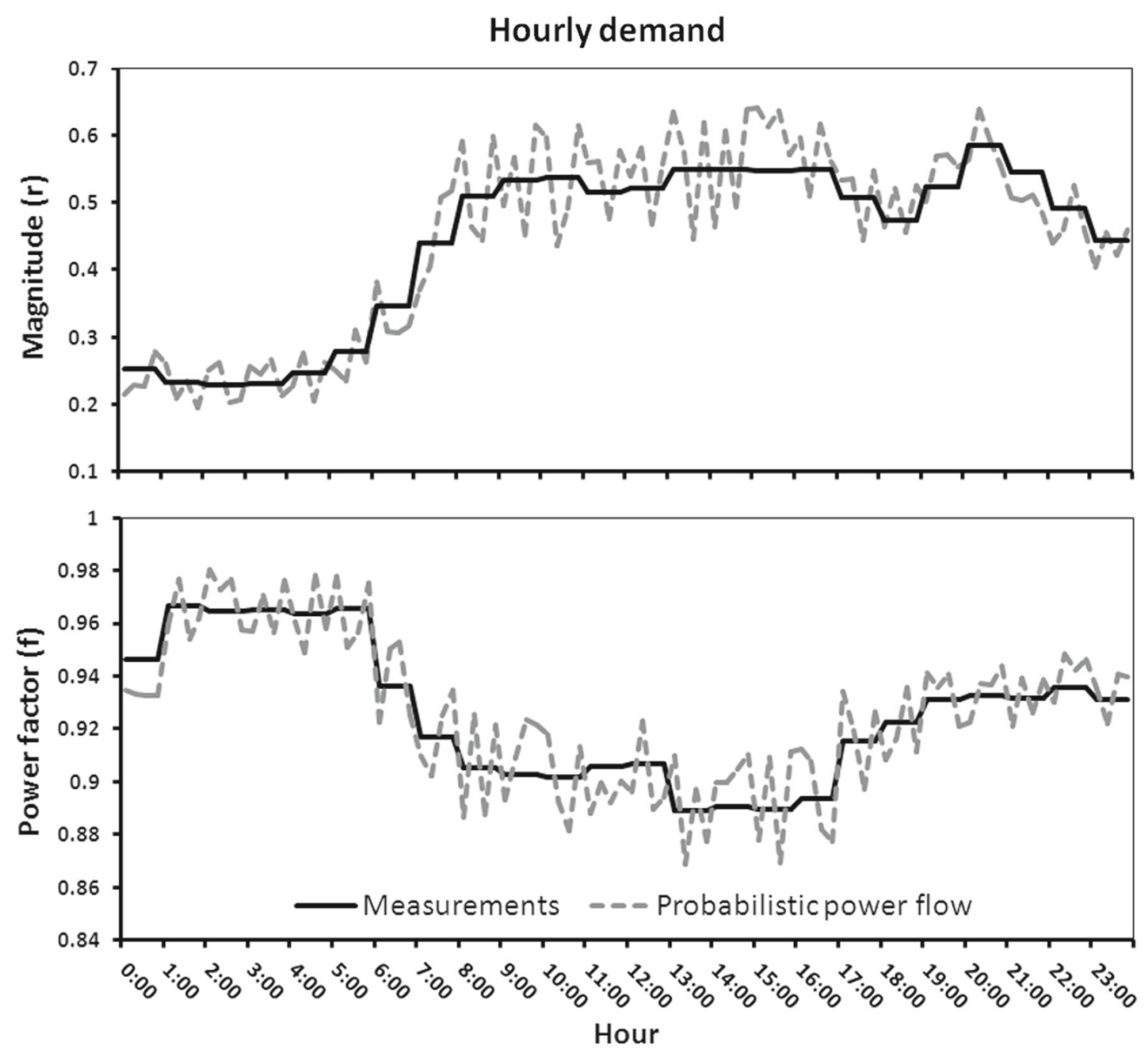

Fig. 13 Hourly power demand of the load \#001

or distortion. The noise figure involves statistical and unpredictable perturbations, while the distortion figure is a fixed operation applied to the signal that can be corrected (Shannon 1984). In this way, the channel is accepted as noisy whenever the data transmission fails due to a noise level greater than a superior limit, which is established according to the type of the employed technology.

$\psi_{i}^{t}\left(t_{s}\right)= \begin{cases}\psi_{i}\left(t_{s}\right)\left[-\frac{N_{0}^{2} A^{4} T_{b}^{2} \ln \left(\gamma_{i}\right)}{2}\right]^{\frac{1}{2}} \leq \frac{A^{2} T_{b}}{2} \\ \varnothing \quad \text { otherwise }\end{cases}$

The communication channel is emulated by (26), where $\psi_{i}^{t}\left(t_{s}\right)$ is the set of electrical parameters transmitted to the system layer and is empty when the maximum noise limit is violated, that is, the metering data are lost and nothing is transmitted to the system layer $\left(\psi_{i}^{t}\left(t_{s}\right)=\emptyset\right)$ whenever the noise energy is greater than half of energy needed to transmit one bit. The first member of the non-strict inequality refers to the inverse Gaussian function, where $\gamma_{i}$ is a random real number, in the range from 0 to 1 , that allows for calculation of the noise level; moreover, $T_{b}$ is the bit duration, $A$ is the bit amplitude, and $N_{0}$ is the noise spectral density.

\section{Partial Results and Discussion}

The proposed methodology was evaluated over a real distribution network that provides energy to 781 loads at the LV network and to 53 loads at the MV network; consequently, there are 47 distribution transformers in the test distribution network. Figure 10 shows a topologic diagram, which shows all distribution transformers and loads at the MV network, whereas only one LV network is shown, which has the loads numbered from \#826 to \#834. Moreover, four important points are highlighted for the analysis: the circuit breaker 


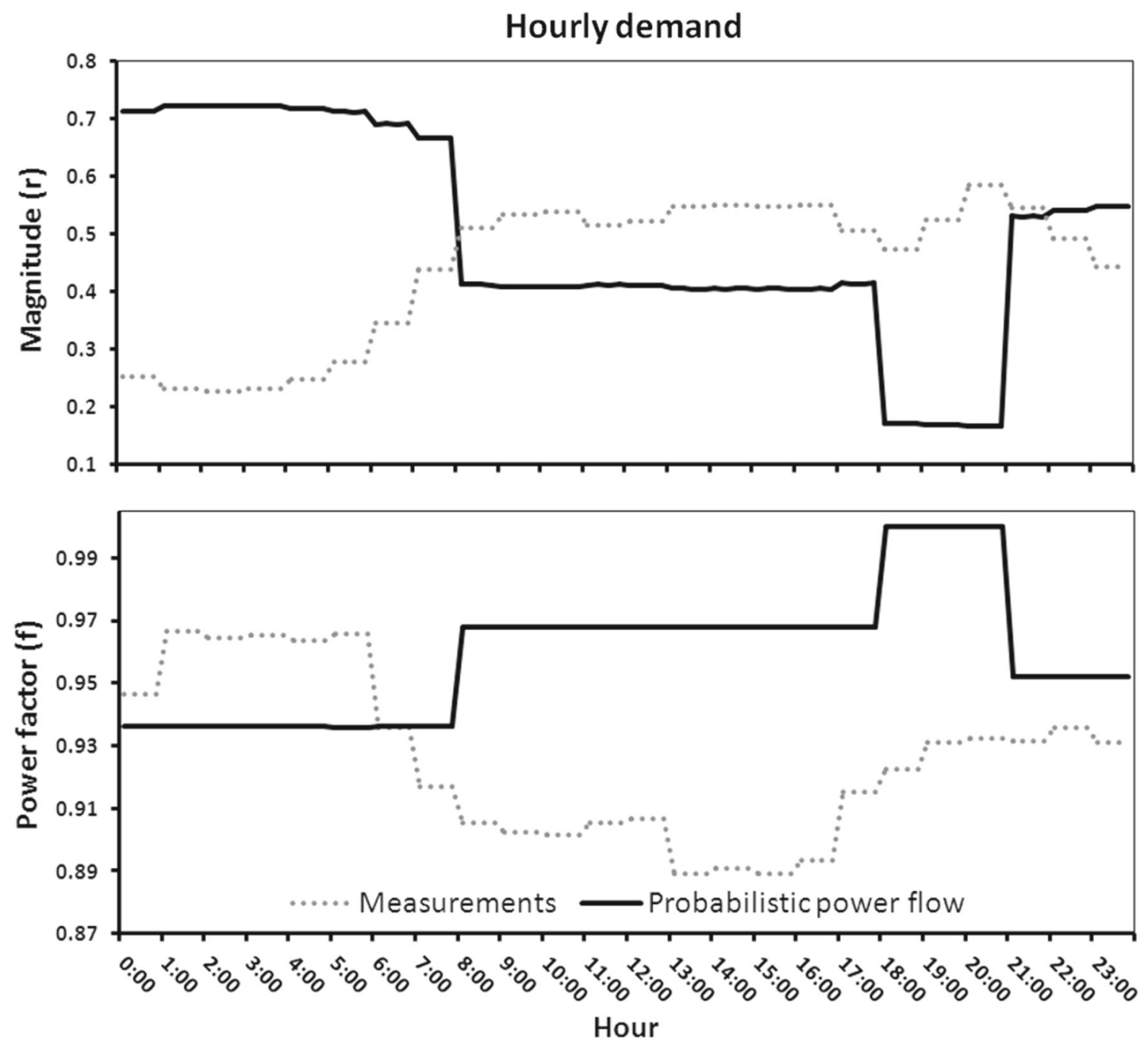

Fig. 14 Hourly power demand of the load \#042

indicates the feeder beginning, load \#001 is near the main power source, load \#042 is influenced by the market, and load \#832 is connected to the LV network.

\subsection{Performance of the Probabilistic Power Flow}

Regarding the evaluation of the methodology proposed by this paper, only the results of phase 'a' are shown because the results of phases, 'b' and 'c' were similar. The evaluation was started by the construction of the voltage profile chart along the network section that begins in the circuit breaker and ends in the load \#832. The presentation of the voltage profile aims to validate the model of the series-connected devices, especially the transformer distribution model. Moreover, the voltage profile chart provides the voltage drop for each bus of the distribution network that is useful during the evaluation of the load model.

The voltage profile calculated by the power flow algorithm is shown in Fig. 11, where the line chart, which has vertical axis scale in kilovolts $(\mathrm{kV})$, highlights the performance of the distribution transformer model that attenuated the voltage from thousands of volts to some hundreds of volts. The simulation also achieved a transformation ratio of approximately 63 that matched the expected ratio once the operating voltage at the MV network was of $13.8 \mathrm{kV}$ and of $220 \mathrm{~V}$ at the LV network. In contrast, the line chart, which has vertical axis scale in pu (per-unit), reveals the voltage drop for the minimized behavior of the distribution transformer. In this way, the voltage of the load \#001 was found to be approximately $1 \mathrm{pu}$, and the voltage of the load \#832 exhibited a decrease of $9 \%$. These results are important to the understanding of the load models that will be discussed in the following paragraphs.

The bus of load \#001 is an ideal point to the assessment of the dependence of the load model upon the factor $\omega$ because, at this bus, the voltage influence on the ZIP load model is minimized. Thereby, the power demand of the load \#001 was simulated at noon for 100 days to build the cumulative 


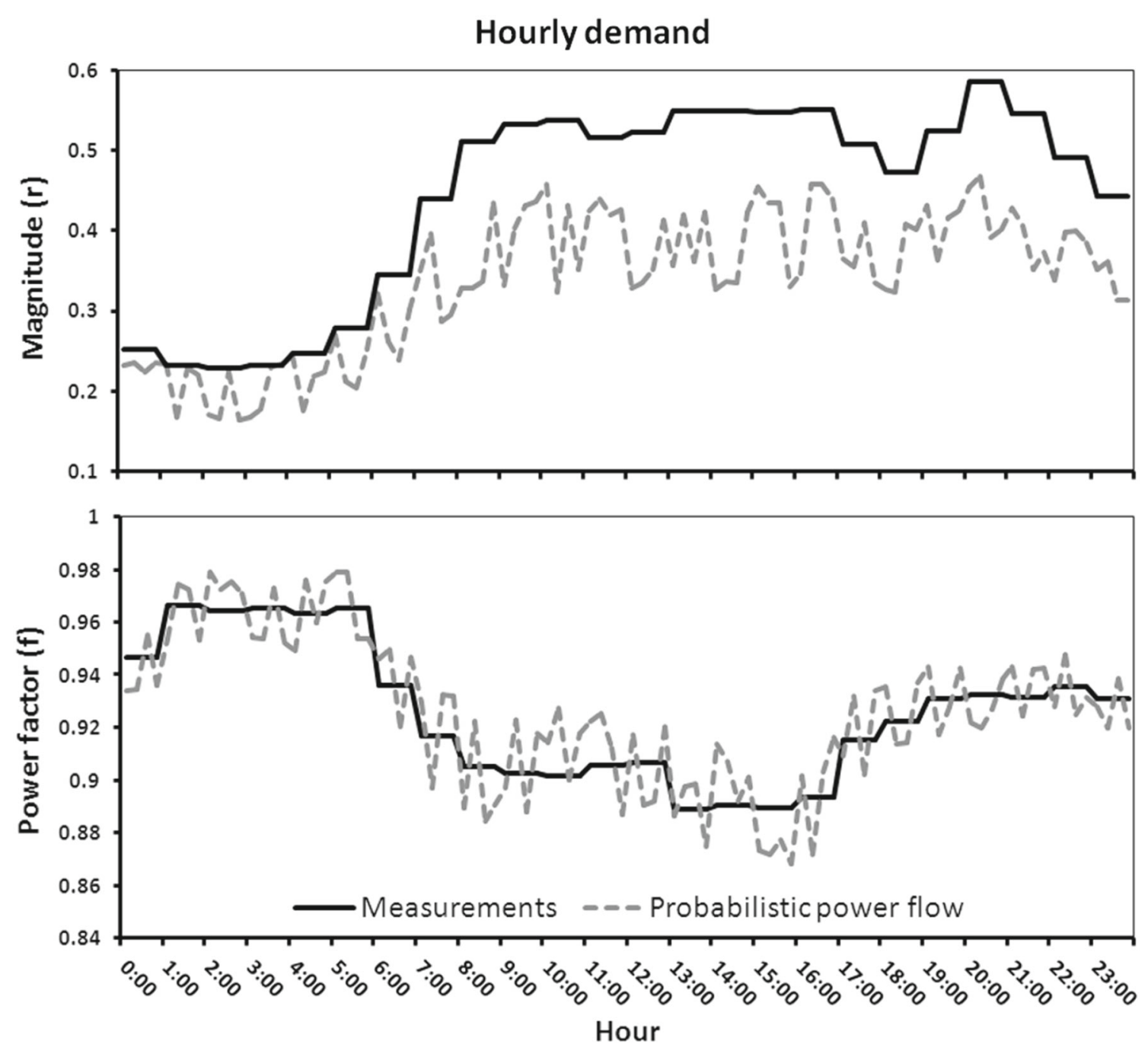

Fig. 15 Hourly power demand of the load \#832

frequency curves, as is presented in Fig. 12. The theoretical curve was built by (13), where the mean and standard deviation were computed using the measurement data. The curves for $\omega=0.7, \omega=0.8$, and $\omega=0.9$ were obtained using the simulation results. The cumulative frequency curve for $\omega=$ 0.7 was closer to the theoretical curve because the fluctuation range was large; however, this large fluctuation range must be avoided once the measurement behavior is lost; as a result, in this paper, the value of $\omega$ equal to 0.8 was used to obtain the power demand.

The ZIP load model also depends to the weight factors, $\lambda_{q}$, that were adjusted with values: $\lambda_{0}=0$ and $\lambda_{1}=\lambda_{2}=0.5$. Subsequently, the power demand of the load \#001 was reproduced using a discrete time interval equal to $15 \mathrm{~min}$, during 24 h. The results are shown in Fig. 13, where the measurement sets $\left(R_{k}\left(t_{h}\right)\right.$ and $\left.F_{k}\left(t_{h}\right)\right)$ are the solid lines, and the results of the probabilistic power flow are the dashed lines. First, a fluctuation was observed of the values of magnitude and power factor for each quarter hour; second, the results obtained by the probabilistic power flow surrounded the measurements of the magnitude and the power factor. These results, fortunately, corresponded to the expected load behavior; hence, the simulator can reproduce the power demand fluctuation in a realistic way.

The market influence on the power demand was evaluated assuming that the load \#042 had a HEMS, and the energy cost fluctuations were according to the chart in Fig. 7. Moreover, parameters were adjusted to $r_{0}=0.95$ and $\delta_{R}=0.75$ for the adopted function of the magnitude controller as well as $f_{0}=0.92$ and $\delta_{F}=0.08$ for the adopted function of the power factor controller. The collected results of the hourly power demand of the load \#042 are shown in Fig. 14, where the fluctuations of the power factor obtained by the probabilistic power flow were directly proportional to the energy cost. In contrast, the fluctuations of the normalized magnitude were inversely proportional to the energy cost because the smart load demands a large amount of power when the total demand of distribution network and the energy cost are low; in addi- 
Fig. 16 Quantization error along MV network

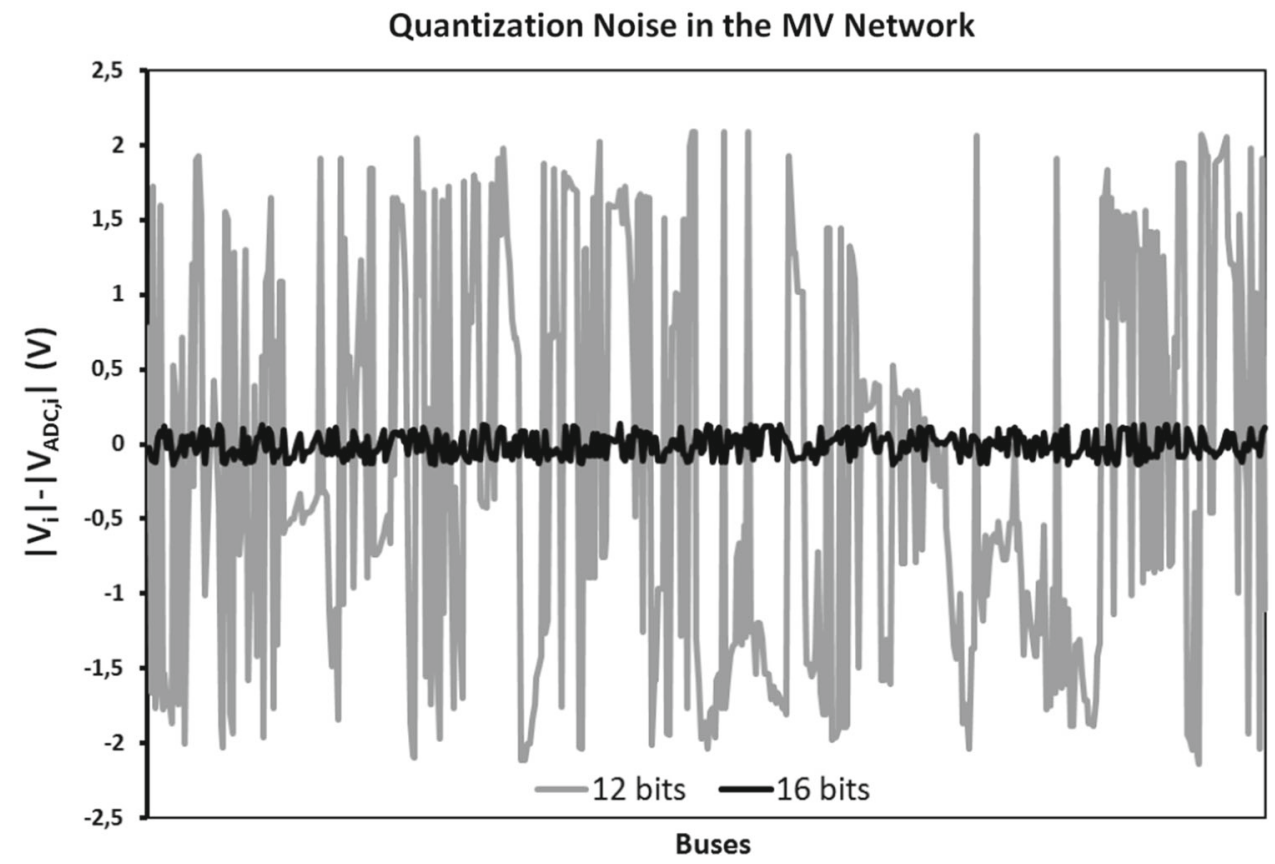

tion, the smart load attempts to compensate the power factor of the distribution network when the total demand of distribution network and the energy cost are high.

The last evaluation point is the bus of the load \#832 that is connected to LV network and had a significant voltage drop of approximately $9 \%$. Figure 15 reveals the hourly power demand of the load \#832, where the measurements of the power factor were surrounded by the fluctuation range of power factor obtained by the probabilistic power flow. However, the fluctuation range of the magnitude obtained from the probabilistic power flow was slightly below the measurements of magnitude, showing the dependence of the ZIP load model upon the voltage drop, which is an indispensable characteristic to reproduce the short-circuit behaviors in a distribution network.

\subsection{Measurement of the Quantization Error}

The voltage profile is an important electrical parameter of power system. The quantization error of the voltage was metered to the analog-digital conversion of voltage using the devices of 12 and 16 bits of resolution, as shown in Fig. 16.

The quantization noise was measured along the MV network, whence the root mean square (RMS) of the noise value was $d V_{R M S, 12}=1.34 \mathrm{~V}$ for 12 bits and $d V_{R M S, 16}=0.07 \mathrm{~V}$ for 16 bits of precision; in percentage values, the voltage differences were $d V_{R M S, 12}=0.017 \%$ and $d V_{R M S, 16}=0.001 \%$, for 12 bits and 16 bits, respectively. The modern PMU has 16 bits of precision and, according the results, the measured quantization error is nearly insignificant, but it must be considered in the posterior analysis.
Table 2 Parameters of the communication channels

\begin{tabular}{lll}
\hline Channel & Error probability & Bandwidth $(\mathrm{Hz})$ \\
\hline Copper cable & $10^{-6}$ & $30 \times 10^{6}$ \\
Microwave & $10^{-4}$ & $40 \times 10^{6}$ \\
Fiber-optic cable & $10^{-12}$ & $4.38 \times 10^{12}$ \\
\hline
\end{tabular}

\subsection{Communication Channel Parameters}

The assessment of the communication channels was performed by the error probability where, initially, typical values of the bit error rate (BER) were assumed for three different channels: microwave, copper cable and fiber-optic cable. Table 2 summarizes the parameters of the communication channels required for the determination of the information transmission.

\section{Conclusions}

The DMS simulation platform proposed in this paper must enable the simulation of the distribution network behaviors and provide an alternative way to develop the resources of operation, planning and control of the smart grid. In part I of this work, three layers of the reference model for the smart grid were mathematically formulated.

The simulator of the physical layer utilized a probabilistic power flow algorithm and was evaluated through a real distribution network, where the power demand fluctuation was analyzed in three different points of the network. Thus, it was verified that the better fluctuation range occurs when $\omega$ is equal to 0.8 , the loads with HEMS are highly dependent 
on the energy cost and the load model enables the simulation of the short-circuit behaviors in the distribution network.

In addition to the provision of the load fluctuations over time, the proposed methodology provides numerous amounts of measuring points in the distribution network, where each point has a unique behavior. The varieties of the load behaviors are ideal for the study of new tools that can be used towards the operation of the modern distribution system. For example, in Leite and Mantovani (2013) a state estimator algorithm was developed and evaluated that should work at the analysis layer from the reference model of the smart grid.

Another important aspect is the simulation of metering interval; in this paper, the metering interval used was a period of just one quarter hour, but the metering interval can be adjusted according to the operator requirements; hence, the metering interval of the algorithm can be specified on a scale of minutes to hours.

The interface and communication layers were also formulated and evaluated through the real distribution network. The results demonstrated the behavior of the quantization noise and the error probability of the communication channels. In this way, part II of this work will investigate the development of the other four upper layers, starting with the system layer.

Acknowledgments This work was fully supported by the Fundação de Amparo à pesquisa do Estado de São Paulo - FAPESP (Grant: 2010/07757-1, 2013/23590-8) and CNPq (Grant 305371/2012-6).

\section{References}

Alhajri, M. F., \& El-Hawary, M. E. (2010). Exploiting the radial distribution structure in developing a fast and flexible radial power flow for unbalanced three-phase networks. IEEE transactions on Power Delivery, 25(1), 378-389.

Byun, J., Hong, I., Kang, B., \& Park, S. (2011). A smart energy distribution and management system for renewable energy distribution and context-aware services based on user patterns and load forecasting. IEEE Transactions on Consumer Electronics, 57(2), 436-444.

Cespedes, R. (2012). A Reference Model for the Electrical Energy System Based On Smart Grids. IEEE T\&D LA 2012, Montevideo.

Cheng, C. S., \& Shirmohammadi, D. (1995). A three-phase power flow method for real-time distribution analysis. IEEE Transactions on Power Systems, 10, 671-679.

Cho, H. S., \& Yamazaki, T. (2009). Determining location of appliances from multi-hop tree structures of power strip type smart meters. IEEE Transactions on Consumer Electronics, 55(4), 2314-2322.

Deilami, S., Masoum, A. S., Moses, P. S., \& Masoum, M. A. (2011). Real-time coordination of plug-in electric vehicle charging in smart grid to minimize power losses and improve voltage profile. IEEE Transactions on Smart Grid, 02(3), 456-467.

Dutta, P., Guan, Yufan, \& Kezunovic, M. (2008). Use of substation IED data for improved alarm processing and fault location. In: Proceedings of the IEEE 40th North American Power Symposium, Calgary.

EPRI (2009). Report to NIST on the Smart Grid Interoperability Standards Roadmap. Contract no. SB1341-09-CN-0031.

Grainger, J. J., \& Civanlar, S. (1985). Volt/Var control on distribution systems with lateral branches using shunt capacitors and voltage regulators. IEEE Transactions on Power Apparatus and Systems, PAS-104(11), 3278-3283.

Granlund, R., Berglund, E., \& Eriksson, H. (2000). Designing webbased simulation for learning. Future Generation Computer System, 17(2), 171-185.

Jeruchim, M. C., Balaban, P., \& Shanmugan, K. S. (2002). Methods of performance evaluation. In Simulation of Communication Systems: Modeling, Methodology and Techniques 2nd (ed.), (pp. 112). Philip Drive Norwell, MA: Kluwer Academic Publishers.

Kanchev, H., Lu, D., Colas, F., Lazarov, V., \& Francois, B. (2011). Energy management and operational planning of a microgrid with a PV-based active generator for smart grid application. IEEE Transactions on Industrial Electronics, 58(10), 4583-4592.

Katiraei, F., Iravani, R., Hatziargyriou, N., \& Dimeas, A. (2008). Microgrids management. IEEE Power and Energy Magazine, 6(3), 5465.

Khushalani, S., Solanki, J. M., \& Schulz, N. N. (2007). Development of three-phase unbalanced power flow using PV and PQ models for distributed generation and study of impact of DG models. IEEE Transactions on Power Systems, 22(03), 1019-1025.

Lee, G. M., \& Su, D. H. (2013). Standardization of smart grid in ITU-T. IEEE Communication Magazine, 51(1), 90-97.

Leite, J. B., \& Mantovani, J. R. S. (2013). State estimation of distribution networks through the real-time measurement of the smart meters. IEEE Grenoble PowerTech, 2013, 1-6.

Leon, R. A., Vittal, V., \& Manimaran, G. (2007). Application of sensor network for secure electric energy infrastructure. IEEE Transactions on Power Delivery, 22(2), 1021-1028.

Lightner, E. M., \& Widergren, S. E. (2010). An orderly transition to a transformed electricity system. IEEE Transactions on Smart Grid, 1(1), 3-10.

Mohsenian-Rad, A. H., \& Leon-Garcia, A. (2011). Distributed internet based load altering attacks against smart power grids. IEEE Transactions on Smart Grid, 2(4), 667-674.

Montenegro, D., Hernandez, M., \& Ramos, G. A. (2012). Real Time OpenDSS framework for Distribution Systems Simulation and Analysis, IEEE T\&D LA 2012, Montevideo.

Niyato, D., \& Wang, P. (2012). Cooperative transmission for meter data collection in smart grid. IEEE Communication Magazine, 50(4), 90-97.

Palensky, P., \& Dietrich, D. (2011). Demand side management: Demand response, intelligent energy systems, and smart loads. IEEE Transactions on Industrial Informatics, 7(3), 381-388.

Qiu, M., Gao, W., Chen, M., Niu, J.-W., \& Zhang, L. (2011). Energy efficient security algorithm for power grid wide area monitoring system. IEEE Transactions on Smart Grid, 2(4), 715-723.

Shannon, C. E. (1984). Communication in the presence of noise. Proceedings of the IEEE, 72(9), 1192-1201.

Shawkat Ali, A. B. M. (2013). Smart Grids: opportunities, developments and trends. In Smart Meter, (pp. 109-133). London: Springer.

Singh, N., Kliokys, E., Feldmann, H., Kussel, R., Chrustowski, R., \& Joborowicz, C. (1998). Power system modeling and analysis in a mixed energy management and distribution management system. IEEE Transactions on Power Systems, 13(3), 1143-1149.

STMicroelectronics (2012), Smart grid distribution and smart meters, [Online] Available: http://www.st.com/st-web-ui/static/active/en/ resource/sales_and_marketing/promotional_material/brochure/br meter.pdf. Accessed 28 July 2014.

Sucic, S., Dragicevic, T., Capuder, T., \& Delimar, M. (2011). Economic dispatch of virtual power plants in an event-driven serviceoriented framework using standards-based communications. Electrical Power System Resource, 81(12), 2108-2119.

zu Eissen, S. M., \& Stein, B. (2006). Realization of web-based simulation services. Elsevier Computers in Industry, 57(3), 261-271. 